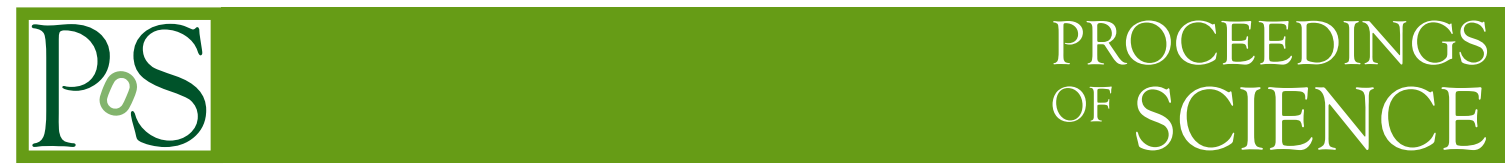

\title{
Status of the ATLAS detector
}

\section{Oleg Solovyanov*}

On behalf of the ATLAS collaboration

State research center Institute for High Energy Physics, Protvino, Russia

E-mail: Oleg. Solovyanov@ihep.ru

\begin{abstract}
After the successful data taking year of 2011 at $7 \mathrm{TeV}$, since March of 2012 the ATLAS experiment at LHC is collecting data with colliding proton beams at the previously unparalleled centre of mass energy of $8 \mathrm{TeV}$. A challenging task was to cope with the augmented event rates due to the increased luminosity delivered by the collider and large pileup conditions. A status of the ATLAS detector is presented as of November 2012. Individual sub-detector systems and their operation status are presented in this paper, together with the main physics results that demonstrate the performance of the detector.
\end{abstract}

LHC on the March - IHEP-LHC,

20-22 November 2012

Institute for High Energy Physics, Protvino,Moscow region, Russia

${ }^{*}$ Speaker. 


\section{Introduction}

The ATLAS detector [1] at LHC [2] had collected data at $7 \mathrm{TeV}$ during the year 2011 run and since March of 2012, and at the time of the presentation, was successfully collecting new statistics with colliding proton beams at the increased centre of mass energy of $8 \mathrm{TeV}$. Compared to the year 2011 data taking, one of the new and challenging tasks was to cope with the augmented event rates due to the increased luminosity delivered by the collider. The other task was to provide the required detector performance in large pile-up conditions. This paper describes the current (November 2012) status of the detector and individual sub-detector statuses together with their performance. The main physics results, obtained with the improved statistics, and plans for the next long shutdown are also the subject of this paper.

\section{ATLAS detector}

Being one of the two general purpose experiments at the LHC, the ATLAS collaboration consists of more than 3000 physicists from 186 institutions of 46 countries, and it celebrated already 20 years of fruitful work, spanning from letter of intent in year 1992 to the experiment approval in 1996, detector installation in 2008, first particle beam collisions in 2009 and, finally, data taking at $7 \mathrm{TeV}$ in 2011 and at $8 \mathrm{TeV}$ in 2012.

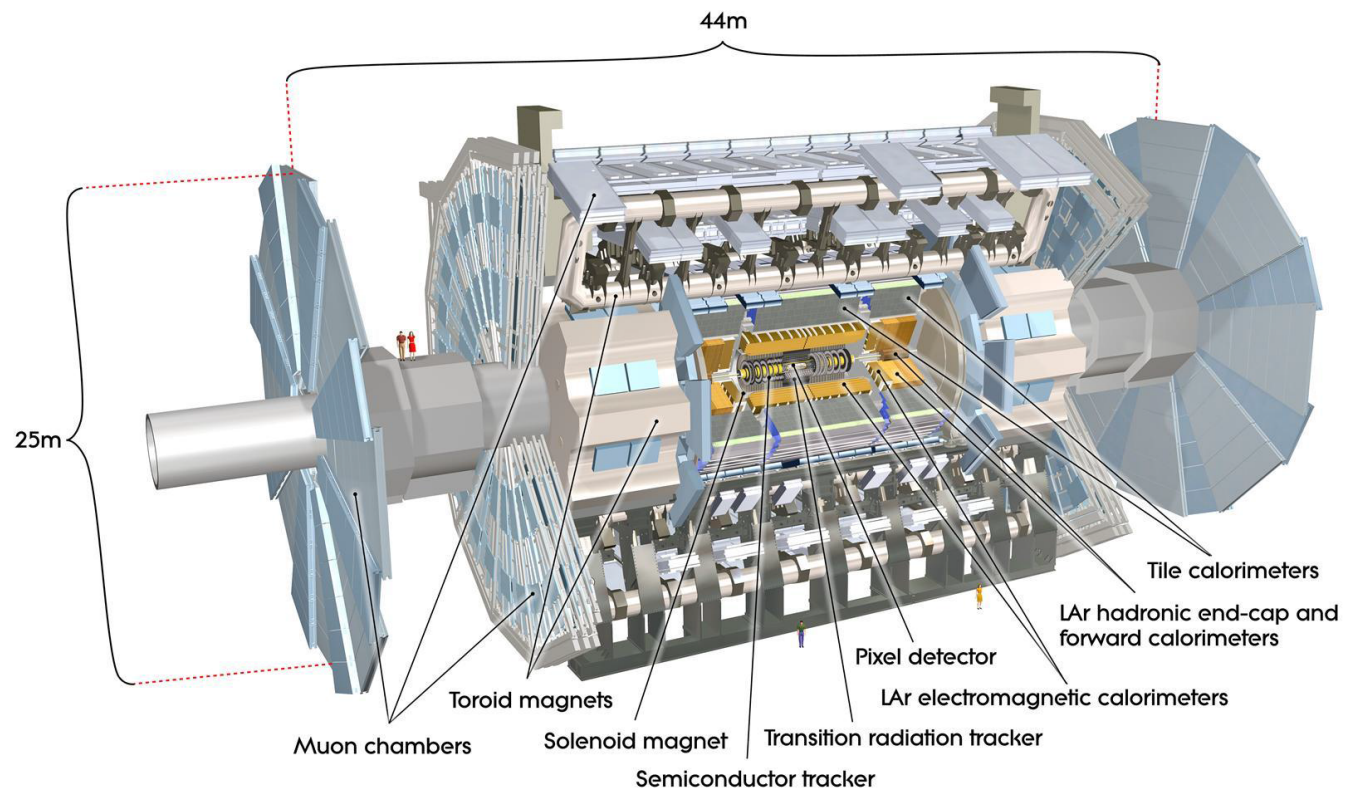

Figure 1: A computer-generated image of the ATLAS detector and it's systems.

The ATLAS detector, schematically shown at Fig. 1, with its length of 46 meters, 12 meter radius, 7000 tons weight and more than 100 millions of readout channels, is currently one of the biggest detectors in the world. The ATLAS sensitivity to a wide spectra of signatures and physics 


\begin{tabular}{lcr}
\hline Subdetector & Number of channels & Approximate operational fraction (\%) \\
\hline Pixels & $80 \mathrm{M}$ & 95.0 \\
SCT Silicon Strips & $6.3 \mathrm{M}$ & 99.3 \\
TRT Transition Radiation Tracker & $350 \mathrm{k}$ & 97.5 \\
LAr EM Calorimeter & $170 \mathrm{k}$ & 99.9 \\
Tile Calorimeter & 9800 & 98.3 \\
LAr Hadronic Endcap Calorimeter & 6500 & 99.6 \\
LAr Forward Calorimeter & 3500 & 99.8 \\
LVL1 Calo trigger & 7160 & 100 \\
LVL1 Muon RPC trigger & $370 \mathrm{k}$ & 100 \\
LVL1 Muon TGC trigger & $320 \mathrm{k}$ & 100 \\
MDT Muon Drift Tubes & $350 \mathrm{k}$ & 99.7 \\
CSC Cathode Strip Chambers & $31 \mathrm{k}$ & 96.0 \\
RPC Barrel Muon Chambers & $370 \mathrm{k}$ & 97.1 \\
TGC Endcap Muon Chambers & $320 \mathrm{k}$ & 98.2 \\
\hline
\end{tabular}

Table 1: Fraction of operational channels in the ATLAS detector for the year 2012 run [3].

is achieved with the help of forward-backward symmetric and cylindric geometry and Inner Detector, Calorimeter system and Muon Spectrometer. The Inner Detector consists of a high precision silicon pixel detector (Pixel) surrounded by a silicon microstrip detector (SCT) and a straw-tube transition radiation tracker (TRT). The Inner Detector lies inside a $2 \mathrm{~T}$ magnetic field provided by a superconducting solenoid. The high-granularity sampling electromagnetic calorimeter is based on liquid argon technology (LAr). A sampling iron-scintillator hadron calorimeter (Tile) is covering the central rapidity range. The Muon Spectrometer that surrounds the calorimeters consists of large air-core superconducting magnets providing a toroidal field in which lies a system of precision tracking (MDT) and fast trigger (RPC, TGC) chambers. The combination of these systems provides charged particle reconstruction together with precise lepton and photon identification, and efficient jet and missing transverse energy reconstruction. To be able to record all possible information and not to loose anything of significance it is important to keep the percentage of the working number of channels as high as possible. Table 1 shows that thanks to the efforts of detector and maintenance teams the fraction of operational channels during 2012 run was more than $95 \%$, and very close to $100 \%$ in most of the sub-systems.

\section{Data taking and trigger}

The LHC performance during the 2012 year run was very stable and strong, with more than $21 \mathrm{fb}^{-1}$ delivered to the experiments (Fig. 2). The peak luminosities routinely reached $7.5 \times 10^{33}$ $\mathrm{cm}^{-2} \mathrm{~s}^{-1}$ as shown in Fig. 3. The number of interactions per bunch crossing during the fill regularly had a peak value of up to 35 and beyond (Fig. 4, 5), while the LHC design figure was 19.

The overall ATLAS data taking efficiency in 2012 at the time of the presentation was $93.7 \%$, similar to the 2011 number. The dead time was the dominant inefficiency source, with important contributions from explicit holding of the trigger during recoveries of the malfunctioning parts of the detector. However, efficient implementations of the automatic recoveries, parallel recovery 


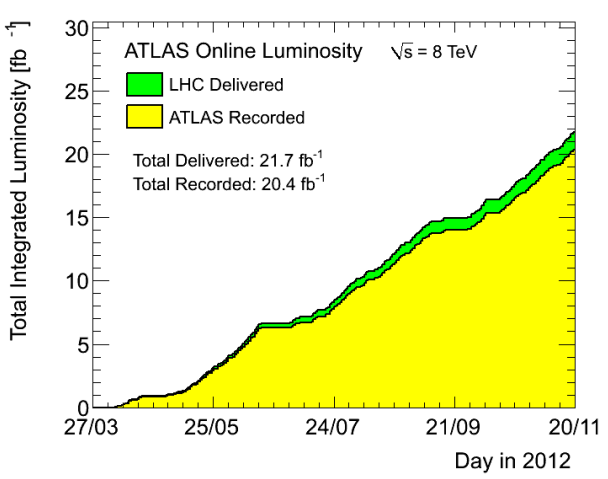

Figure 2: Cumulative luminosity versus day delivered to (green), and recorded by ATLAS (yellow) during stable beams and for pp collisions at $8 \mathrm{TeV}$ centre-of-mass energy in 2012 [4].

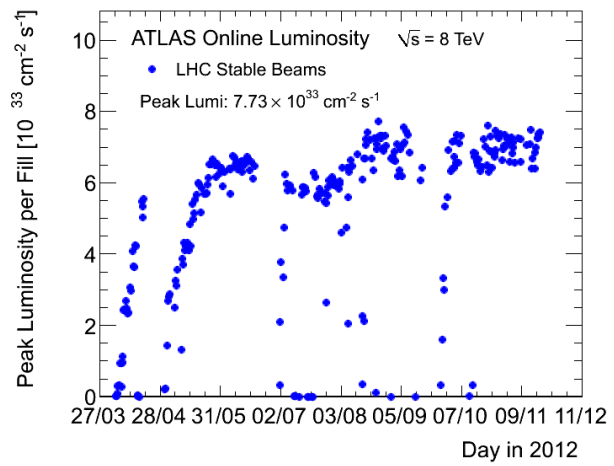

Figure 3: The maximum instantaneous luminosity versus day delivered to ATLAS [4]. Only the peak luminosity during stable beam periods is shown.

mechanisms, knowledge of when and what recovery is appropriate, allowed ATLAS to limit the losses.

Because the LHC was running with 50 ns bunch spacing, the detectors experienced double pile-up conditions for same luminosity, with respect to the designed $25 \mathrm{~ns}$ operation. The pileup has to be fought and mitigated at all levels, such as trigger, reconstruction of physics objects, isolation cuts, etc.

The three-layer trigger system with more than 550 items in the trigger menu managed to keep the inclusive unprescaled lepton thresholds within $\sim 5 \mathrm{GeV}$ over the last two years in spite of the factor of $\sim 70$ of the peak luminosity increase.

In 2012 the trigger selection was re-optimised, e.g. adding lepton isolation, to maintain low the unprescaled thresholds, e.g. for inclusive leptons, regardless of the two times higher luminosity and pile-up than in 2011 data taking.

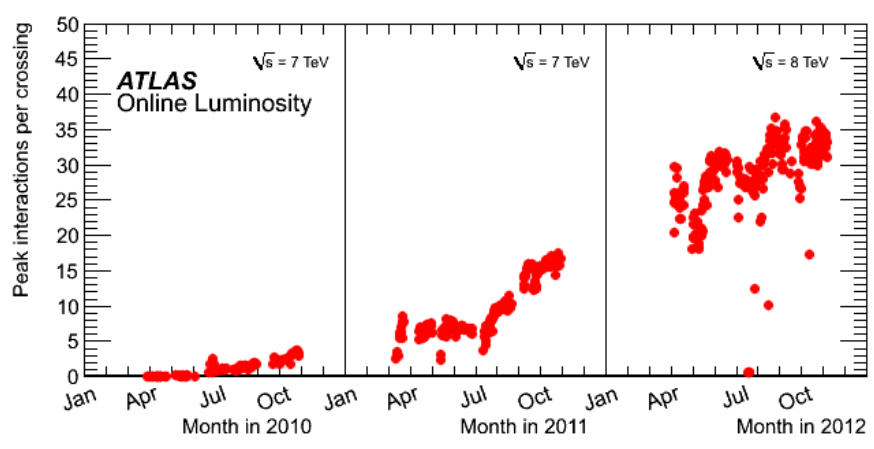

Figure 4: The evolution of the peak number of simultaneous interactions per one bunch crossing during the three data taking periods $(2010,2011,2012)$ [4]. The LHC design figure was 19.

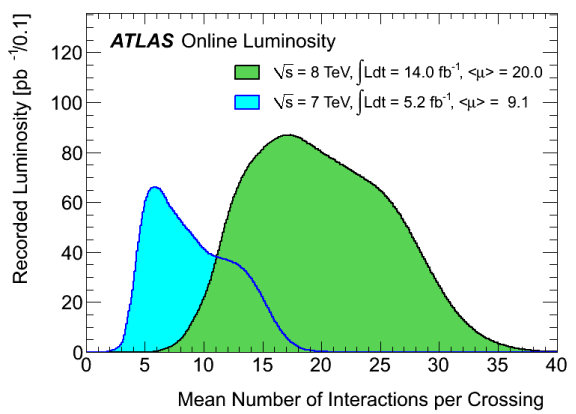

Figure 5: The distribution of the mean number of interactions per bunch crossing for 2011 (blue) and 2012 (green) data taking periods [4].

To cope with this challenging situation, a number of pile-up robust algorithms was developed with almost flat performance as function of pile-up, while keeping the CPU usage under control. 
The results from 2012 operation showed that the trigger was coping very well in terms of rates, efficiencies and robustness with the harsh conditions of 2012 data taking while meeting stringent physics requirements.

\section{Sub-detectors status and performance}

\subsection{Forward detectors}

The ATLAS luminosity monitor detector (LUCID) delivered luminosity measurement since the beginning of the $2012 \mathrm{pp}$ run, but at the highest pile-up had encountered serious problems for event counting due to the saturation and migration effects. One had to integrate the pulse charge over periods of about 1 minute, so that could replace the traditional event counting method. The detector will be taken out for repairs during the forthcoming long shutdown.

The zero-degree calorimeter (ZDC) had suffered heavily from radiation, and was taken out after 2011 run for consolidation work. It had been planned to be ready for HI run, but due to the tight commissioning schedule it might not be possible.

Another ATLAS luminosity detector (ALFA) had successfully took the data at $\beta^{*}=90 \mathrm{~m}$ and $\beta^{*}=1 \mathrm{~km}$ to study elastic and diffractive processes.

\subsection{Inner detectors}

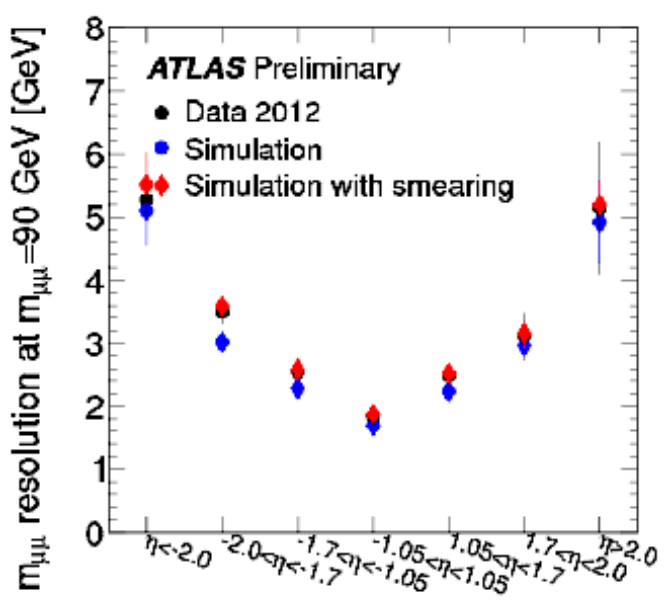

Figure 6: Di-muon mass resolution for muons from $\mathrm{Z}$ boson decays using the momentum measurements in the inner detector [5].

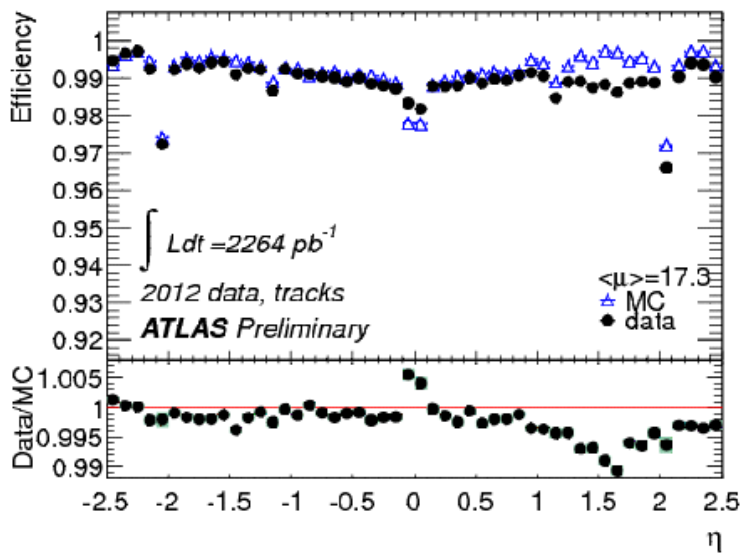

Figure 7: Inner detector reconstruction efficiency versus the muon pseudorapidity in 2012 data and simulation [5].

For the inner tracking detectors the leakage currents were increasing in line with predictions from radiation damage model. The effort was made to further improve operations efficiency and understand the detector behaviours/radiation effects.

To further enhance the performance of the sub-detectors, Pixel will have to reduce the number of Readout Driver (ROD) busies, while SCT still has to improve its module reconfiguration during the run. The TRT should mitigate the gas leaks. 
Despite of these problems, the inner detectors showed excellent performance (Fig. 6) with high reconstruction efficiency (Fig. 7), while simulation had correctly represented the data.

The overall data quality of all inner detectors was about $99 \%$ in 2012 data taking period.

\subsection{Calorimetry}

The LAr electromagnetic calorimeter was very stable, with no major problems in the data taking. The data quality efficiency was all-time high, constantly improving over the course of the run. Most of LAr related inefficiency and small problems will be corrected off-line, during the reprocessing of the data. LAr will be ready to tackle the data taking conditions after the coming long shutdown, for example to read out 4 instead of 5 digitized ADC samples per event, in order to reduce the data bandwidth requirements and processing time.

The Tile hadron calorimeter was performing well, with the number of masked cells close to $2 \%$, mainly due to 4 dead low voltage (LV) front-end power supplies. The Tile data acquisition system had improved drastically the automatic online recovery procedures after the trips of the front-end power supplies, reducing the data losses due to these trips. A number of the production version of the redesigned LV front-end power supply was installed in the cavern before the 2012 run. It proved to be more stable (only one trip was detected) and had lower noise. The sub-detector will be fully equipped with the new version during the next long shutdown.

The electron energy response shows excellent stability with respect to increasing pile-up in 2012 data (Fig. 8). The stability of photon conversion reconstruction is also good (Fig. 9). For the 2012 data taking, the electron reconstruction algorithm has been improved with respect to the 2011 for both data and MC: $\sim 1 \%$ in the barrel region of the calorimeter and $\sim 5 \%$ in the end-caps.

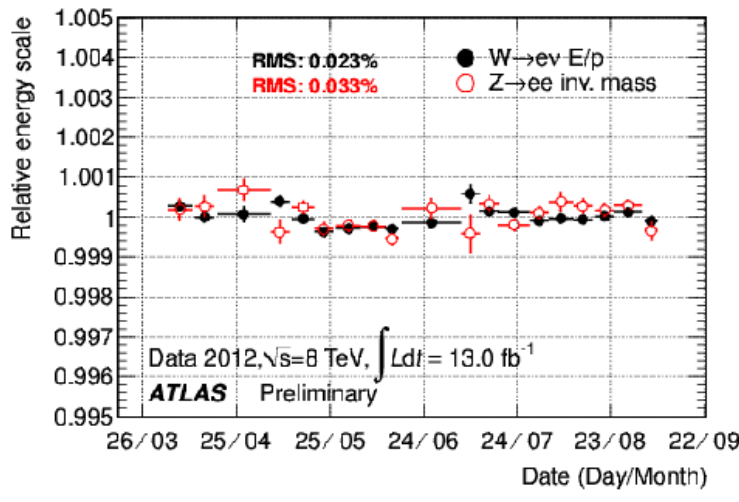

Figure 8: The fitted peak value of the $\mathrm{Z} \rightarrow$ ee invariant mass as well as the most probable value of the E/p distribution from electrons coming from $\mathrm{W} \rightarrow \mathrm{e} v$ decays as a function of time [6].

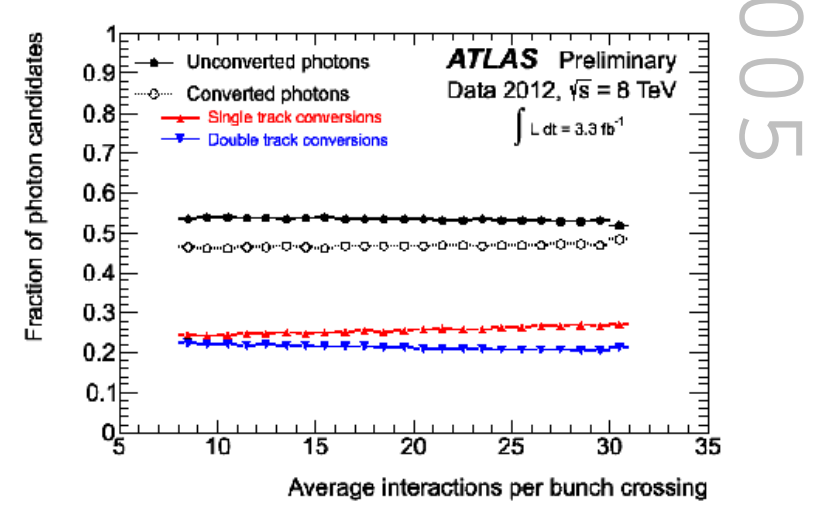

Figure 9: The fraction of unconverted and converted (single and double track conversions) photon candidates as a function of the average number of interactions per bunch crossing [7].

The missing transverse energy reconstruction performed quite well under the complicated pileup conditions of 2012 data. The resolution performance was stable with respect to the increasing pile-up. There were several improvements in pile-up corrections and there was quite a good description of data by simulation. Higher pile-up conditions that are expected after the long shutdown will pose more challenges. 


\subsection{Muon spectrometer}

The muon spectrometer was in good shape with generally smooth running with occasional hiccups. The TGC ROD had improved the auto-recovery while minimizing the downtime. The causes of TGC ROD busies were still being investigated. The alignment of the spectrometer was done with collision data and magnetic field off configuration. There was a good agreement between simulation and data (Fig. 10) and excellent resolution for di-muon measurements with corrections for energy loss in the calorimeters (Fig. 11).

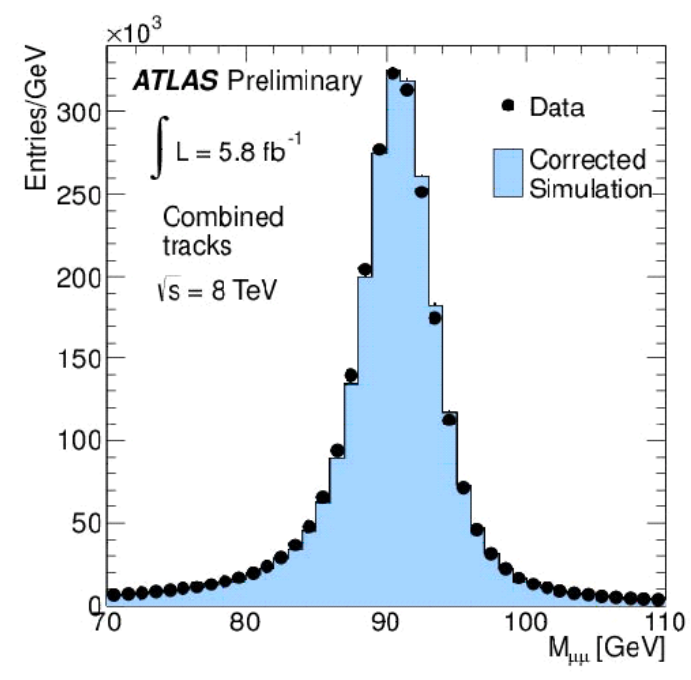

Figure 10: Di-muon invariant mass for combined muons with $\mathrm{p}_{\mathrm{T}}>25 \mathrm{GeV}$ [8]. The plot shows the invariant mass for 2012 data and corrected (smeared momenta) simulation of $\mathrm{Z} \rightarrow \mu \mu+$ backgrounds.

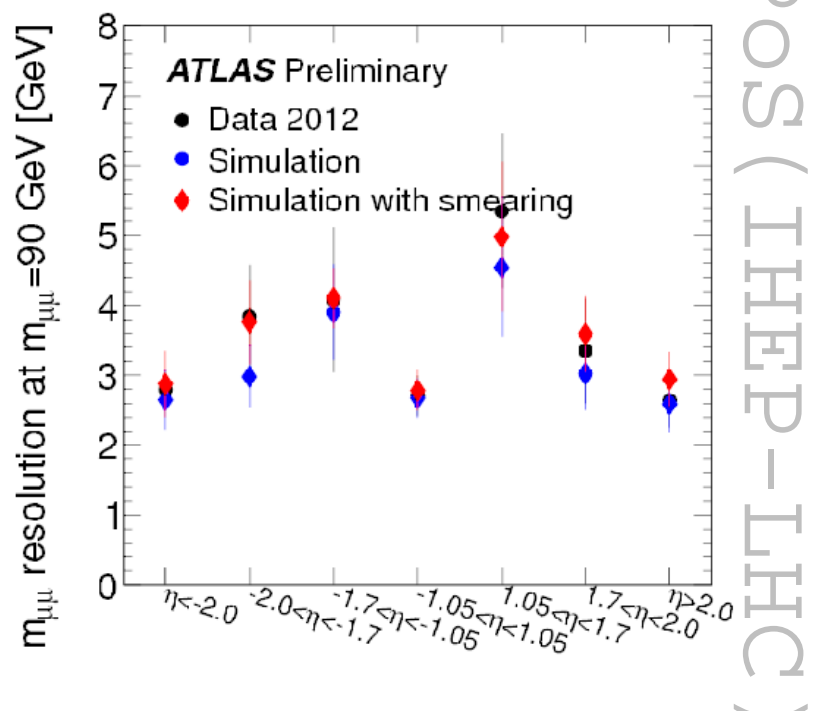

Figure 11: Di-muon mass resolution for muons from $\mathrm{Z}$ boson decays using the momentum measurements in the muon spectrometer corrected for the energy loss in the calorimeters [5].

\section{Physics results}

At the time of the presentation ATLAS had submitted/published more than 400 conference notes and more than 200 physics papers, including the most important one: "First observations of a new particle in the search of the Standard Model Higgs boson at the LHC" [10].

For the Standard Model (SM), the measurements of the cross-sections from inclusive W, Z to $\mathrm{ZZ}$ production were done as the foundation while probing for the new physics, and as a background for new searches and precision measurements (Fig. 12).

The main result was the Higgs significance increase from 5.2 to $5.9 \sigma$ thanks to the inclusion of the results from $\mathrm{H} \rightarrow \mathrm{WW}^{*}$ search for 2012 data (Fig. 13).

ATLAS was also seriously attacking the weak scale SUSY in the region between $100 \mathrm{GeV}$ and $1 \mathrm{TeV}$ with inclusive searches, natural SUSY, long-lived particles and the RPV.

While there were no signs of the new exotic physics at the time of the presentation, ATLAS still had a lot of the $8 \mathrm{TeV}$ analyses in the pipeline, including extra dimension searches, leptoquarks and excited fermions. 
The heavy ions program was ongoing, for the 2012 there were no new $\mathrm{Pb}+\mathrm{Pb}$ results yet, but more than 2 million $\mathrm{p}+\mathrm{Pb}$ events from the pilot run. $\mathrm{The} \mathrm{p}+\mathrm{Pb}$ run will happen in early 2013; and the next $\mathrm{Pb}+\mathrm{Pb}$ data are expected in 2015 .

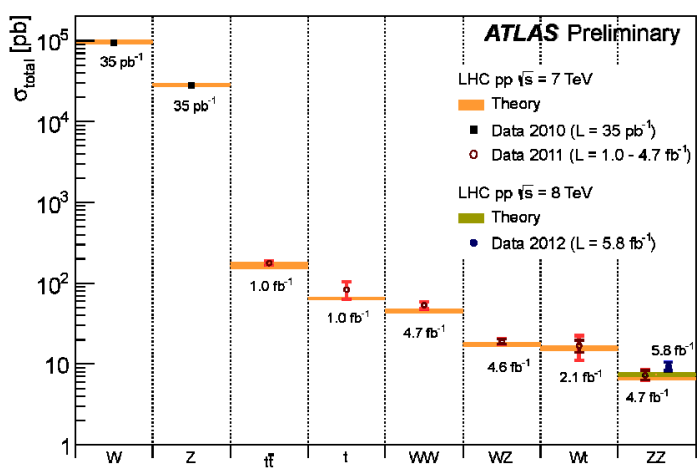

Figure 12: Summary of several Standard Model total production cross section measurements, corrected for leptonic branching fractions, compared to the corresponding theoretical expectations [9].

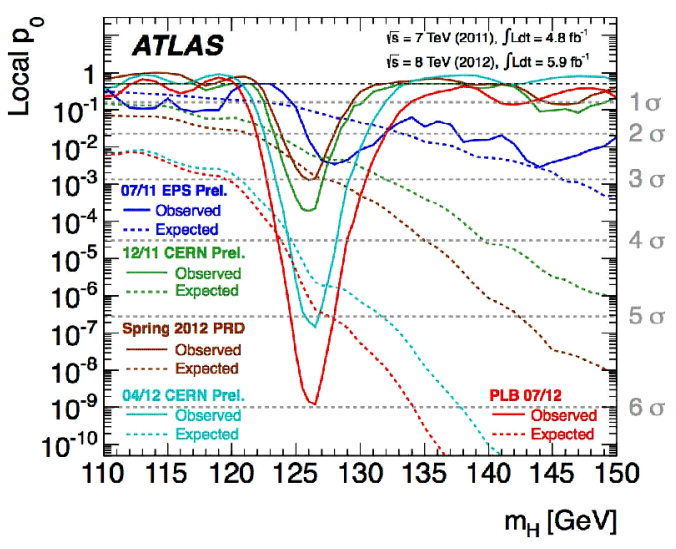

Figure 13: The evolution of the local probability p0 for a background-only experiment to be more signal-like than the observation as a function of Higgs mass [10].

\section{Long shutdown and upgrade plans}

During the coming long shutdown 1 (LS1) most of the sub-detectors will perform the repairs and consolidation of their front-end and back-end electronics.

Also during the LS1 the Phase-0 upgrade is taking place with some major improvements to physics capabilities. The new small Beryllium beam pipe will be installed together with the new insertable pixel b-layer (IBL). The IBL preserves current physics performance at very high pileup conditions of the next data taking periods.

The completion of the installation of the end-cap extra (EE) muon chambers will take place and some additional chambers in the feet and elevator regions will be installed.

During the Phase- 0 upgrade a new part of the trigger system will be introduced: a topological processing at the level 1. And the maximum level 1 trigger accept rate will be increased from 75 up to $100 \mathrm{kHz}$.

\section{Summary}

During the 2012 run the ATLAS experiment successfully recorded more than $20 \mathrm{fb}^{-1}$ of good quality data at $8 \mathrm{TeV}$ with $\sim 94 \%$ efficiency.

The ATLAS detector is performing well, in good agreement with simulation, coping with increasing luminosity and large pile-up conditions using optimized trigger setup.

More than 200 physics papers using collision data have been published, including the most important one: "First observations of a new particle in the search of the Standard Model Higgs boson at the LHC". 
The upgrade $R \& D$ and projects are well advanced and running at full speed in preparation for the long shut down 1.

Many thanks to the LHC machine and all ATLAS collaboration for their amazing performance!

\section{References}

[1] ATLAS Collaboration, The ATLAS Experiment at the CERN Large Hadron Collider, JINST 3 (2008) S08003.

[2] L. Evans and P. Bryant (editors), LHC Machine, JINST 3 (2008) S08001.

[3] https://twiki.cern.ch/twiki/bin/view/AtlasPublic/ApprovedPlotsATLASDetector

[4] https://twiki.cern.ch/twiki/bin/view/AtlasPublic/LuminosityPublicResults

[5] https://atlas.web.cern.ch/Atlas/GROUPS/PHYSICS/MUON/PublicPlots/2012/June

[6] https://atlas.web.cern.ch/Atlas/GROUPS/PHYSICS/EGAMMA/PublicPlots/20121106/ATL-COMPHYS-2012-1593

[7] https://atlas.web.cern.ch/Atlas/GROUPS/PHYSICS/EGAMMA/PublicPlots/20120620/PhotonRecoStability/ATLCOM-PHYS-2012-823/

[8] https://atlas.web.cern.ch/Atlas/GROUPS/PHYSICS/MUON/PublicPlots/2012/ATL-COM-MUON2013-006

[9] https://twiki.cern.ch/twiki/pub/AtlasPublic/CombinedSummaryPlots

[10] ATLAS Collaboration, Observation of a new particle in the search for the Standard Model Higgs boson with the ATLAS detector at the LHC, Phys. Lett. B 716 (2012) 1-29. 